\section{Fatores associados ao aleitamento cruzado em duas cidades do Sudeste do Brasil}

\author{
Factors associated with cross-nursing in two \\ cities in Southeast Brazil
}

\section{Factores asociados a la lactancia materna cruzada en dos ciudades del sudeste de Brasil}

\author{
Mariana Pujól von Seehausen 1 \\ Maria Inês Couto de Oliveira 1 \\ Cristiano Siqueira Boccolini 2 \\ Maria do Carmo Leal 3
}

doi: 10.1590/0102-311X00038516

\section{Resumo}

O objetivo foi estimar a prevalência de aleitamento cruzado em duas cidades brasileiras e analisar os fatores associados à prática. Estudo transversal aninhado a uma coorte de gestantes recrutadas nas unidades públicas de saúde que ofereciam atendimento pré-natal em duas cidades de médio porte do Estado do Rio de Janeiro, Brasil: da Região Metropolitana e da Região Serrana, conduzido entre 2008 e 2010. O presente trabalho inclui todas as mulheres entrevistadas aos seis meses de vida de seus bebês. Razões de prevalência ajustadas foram obtidas por modelo de regressão de Poisson e as variáveis de exposição que alcançaram $p \leq 0,05$ compuseram o modelo final. $O$ aleitamento cruzado foi praticado por 43,4\% das mães na região metropolitana e por 34,5\% delas na Região Serrana. O baixo nível socioeconômico esteve associado ao aleitamento cruzado nas duas cidades. Além disso, na Região Serrana foram associados diretamente ao desfecho ser mãe adolescente, ter escolaridade igual ou inferior ao Ensino Fundamental completo e ter realizado menos de seis consultas pré-natais. Na Região Metropolitana, além do nível socioeconômico, apenas a multiparidade foi associada (inversamente) ao aleitamento cruzado. O aleitamento cruzado, embora contraindicado pelo Ministério da Saúde, apresentou alta prevalência entre as mães entrevistadas e foi mais praticado pelas populações mais vulneráveis.

Aleitamento Materno; Atenção Primária à Saúde; Saúde da Criança

\author{
Correspondência \\ M. P. von Seehausen \\ Programa de Pós-graduação em Saúde Coletiva, Instituto de \\ Saúde Coletiva, Universidade Federal Fluminense. \\ Rua Marques de Paraná 303, anexo, 4o andar, Niterói, RJ \\ 24033-900, Brasil. \\ maripujol@hotmail.com \\ 1 Instituto de Saúde Coletiva, Universidade Federal Fluminense, \\ Niterói, Brasil. \\ 2 Instituto de Comunicação e Informação Científica e \\ Tecnológica em Saúde, Fundação Oswaldo Cruz, Rio de Janeiro, \\ Brasil. \\ 3 Escola Nacional de Saúde Pública Sergio Arouca, Fundação \\ Oswaldo Cruz, Rio de Janeiro, Brasil.
}




\section{Introdução}

O aleitamento cruzado, prática em que uma mulher amamenta o filho de outra mulher, tem raízes históricas, com relatos desde 2000 a.C. 1. No Brasil Colônia, o ato de amamentar era relegado a amas de leite escravas que viviam com a família da criança, que a amamentava e cuidava em uma relação íntima e carinhosa 2. Atualmente, o aleitamento cruzado é percebido como um compartilhamento informal e ocasional entre mães, não remunerado, podendo ser recíproco 3,4,5.

No Brasil, o aleitamento cruzado passou a ser formalmente contraindicado pelo Ministério da Saúde em 1996, devido ao risco de transmissão vertical do HIV pela amamentação 6. Contudo, o Ministério da Saúde não inclui informações sobre a contraindicação ao aleitamento cruzado em materiais institucionais, como a Caderneta da Gestante (http://189.28.128.100/dab/docs/portaldab/documentos/ caderneta_gestante.pdf, acessado em 03/Dez/2015) e a Caderneta da Criança (http://bvsms.saude.gov. br/bvs/publicacoes/caderneta_saude_crianca_5ed.pdf, acessado em 06/Nov/2015). Essa prática não é monitorada nos serviços de saúde e os estudos epidemiológicos relacionados ao tema são escassos 7,8, não sendo possível avaliar como essa informação vem sendo comunicada pelos profissionais de saúde. Um estudo conduzido em Ribeirão Preto, São Paulo, observou que puérperas atendidas em serviço público não tinham informação sobre a contraindicação do aleitamento cruzado 9 .

Foi encontrado apenas um artigo no Brasil sobre a prática do aleitamento cruzado e seus determinantes 8 , sendo necessário analisar este desfecho em outros contextos. Este trabalho teve por objetivo estimar a prevalência de aleitamento cruzado em duas cidades brasileiras e analisar os fatores associados à prática.

\section{Métodos}

Estudo transversal aninhado a uma coorte de gestantes oriundas do atendimento público ao pré-natal em duas cidades de médio porte (150.000 a 300.000 habitantes) do Estado do Rio de Janeiro, denominadas de cidade da Região Metropolitana e cidade da Região Serrana. A coorte original teve como objetivo analisar a associação entre capital social e desfechos perinatais. As duas cidades eram de médio porte e tinham perfis socioeconômicos distintos: a cidade da Região Metropolitana apresentava uma renda per capita 118\% menor e uma taxa de homicídios dez vezes maior do que a cidade da Região Serrana. A cidade da Região Metropolitana contava com uma proporção de ginecologistas por mulher três vezes menor do que a cidade da Região Serrana, indicando também diferenças no perfil de atenção à saúde 10 .

A amostra inicial foi estabelecida em 1.400 mulheres, considerando-se uma prevalência de 25,8\% de pré-natal inadequado e de $8,3 \%$ de pré-natal mais do que adequado, com nível de significância de $5 \%$ e poder de teste de $95 \%$. Foram acrescentados $20 \%$ devido a possíveis perdas de acompanhamento, resultando em uma amostra de 1.680 mulheres, sendo 840 em cada município 10 . Para este trabalho, cujo desfecho é o aleitamento cruzado, para um poder de estudo de $80 \%$, alfa de $5 \%$, prevalência esperada de 40\% (aferida no estudo piloto) e para detectar uma razão de chance de 1,6 seria necessária uma amostra mínima de 576 mulheres em cada cidade.

As mulheres foram recrutadas em cinco unidades públicas de saúde que ofereciam atendimento prénatal (três na cidade da Região Metropolitana e duas na cidade da Região Serrana), que representavam 90\% de todo o atendimento pré-natal público. Foram elegíveis gestantes no primeiro trimestre de gestação. Mulheres que tiveram aborto ou bebês natimortos foram excluídas do estudo. O acompanhamento da coorte foi realizado por monitoramento telefônico, sendo agendadas entrevistas domiciliares durante o seguimento e entrevistas na maternidade no período de pós-parto.

As entrevistas começaram simultaneamente em ambos os municípios em 2008 e terminaram em 2010, sendo realizadas por 14 entrevistadoras treinadas para a aplicação de questionário padronizado. As entrevistadoras permaneciam todos os dias da semana em que havia atendimento pré-natal nas unidades de saúde, entrevistando todas as gestantes até que se atingisse o tamanho amostral. Na entrevista de base houve $4 \%$ de recusas que foram repostas por outras gestantes até que fosse atingido o tamanho amostral de 1.680 .

Foram realizadas entrevistas presenciais em quatro momentos: pré-natal (primeiro trimestre da gestação), pós-parto imediato (até 30 dias após o parto), aos três e seis meses de vida do bebê (com 15 dias 
de tolerância cada). Este estudo incluiu todas as mulheres entrevistadas aos seis meses de vida de seus bebês ( $\mathrm{n}=586$ na cidade da Região Metropolitana e $\mathrm{n}=732$ na cidade da Região Serrana, totalizando $\mathrm{n}=1.318$, ou $78,9 \%$ da amostra inicial).

Na entrevista de base no pré-natal, foram coletados os dados sociodemográficos. No pós-parto, foram coletados dados relativos à história reprodutiva, ao parto, nascimento e características do recém-nascido. Aos seis meses de vida buscou-se informações sobre trabalho materno, viver com companheiro, alimentação dos bebês e sobre o aleitamento cruzado. O aleitamento cruzado foi definido como a mulher amamentar outra(s) criança(s) que não o seu filho e/ou ter o seu filho amamentado por outra(s) mulher(es) 3,4, sendo a não prática de aleitamento cruzado a categoria de referência. Foram incluídas perguntas sobre a relação entre as mães envolvidas e suas motivações para a prática.

O nível socioeconômico foi subdividido nas classes: A (mais alta), B, C, D e E (mais baixa), segundo a Associação Brasileira de Empresas de Pesquisa 11. Para este estudo, foram agrupadas as classes D e E, uma vez que poucos $(5,2 \%)$ se encontravam na classe E, não sendo encontrado nenhum sujeito classificado como classe A. A cor da pele foi autorreferida pelas mulheres, conforme a classificação do Instituto Brasileiro de Geografia e Estatística (IBGE) 12, sendo então dicotomizadas em brancas e não brancas (pretas, pardas, amarelas e indígenas).

A fim de conhecer a distribuição das variáveis de exposição e do desfecho aleitamento cruzado, foi desenvolvida análise univariada. A seguir, foi conduzida uma análise bivariada, utilizando-se o teste qui-quadrado de Pearson, com intervalos de 95\% de confiança (IC95\%), sendo incluídas na modelagem estatística as variáveis que registraram valor de $\mathrm{p} \leq 0,20$.

As razões de prevalência ajustadas foram obtidas pelo modelo de regressão de Poisson com variância robusta, pois o desfecho apresentou uma prevalência elevada ${ }^{13}$. As variáveis de exposição que alcançaram valor de $\mathrm{p} \leq 0,05$ compuseram o modelo final. A regressão seguiu o modelo conceitual hierarquizado, segundo a proximidade temporal de cada variável com o desfecho 14 . Características maternas e contextuais foram consideradas como variáveis distais; características reprodutivas, do pré-natal e parto como variáveis intermediárias, já atividades e hábitos maternos e a assistência da criança foram considerados como variáveis proximais (Figura 1).

O estudo foi aprovado pelo Comitê de Ética em Pesquisa da Escola Nacional de Saúde Pública Sergio Arouca, Fundação Oswaldo Cruz - protocolo no 158/06.

\section{Figura 1}

Modelo teórico da prática de aleitamento cruzado em duas cidades do Estado do Rio de Janeiro, Brasil, 2008-2010.

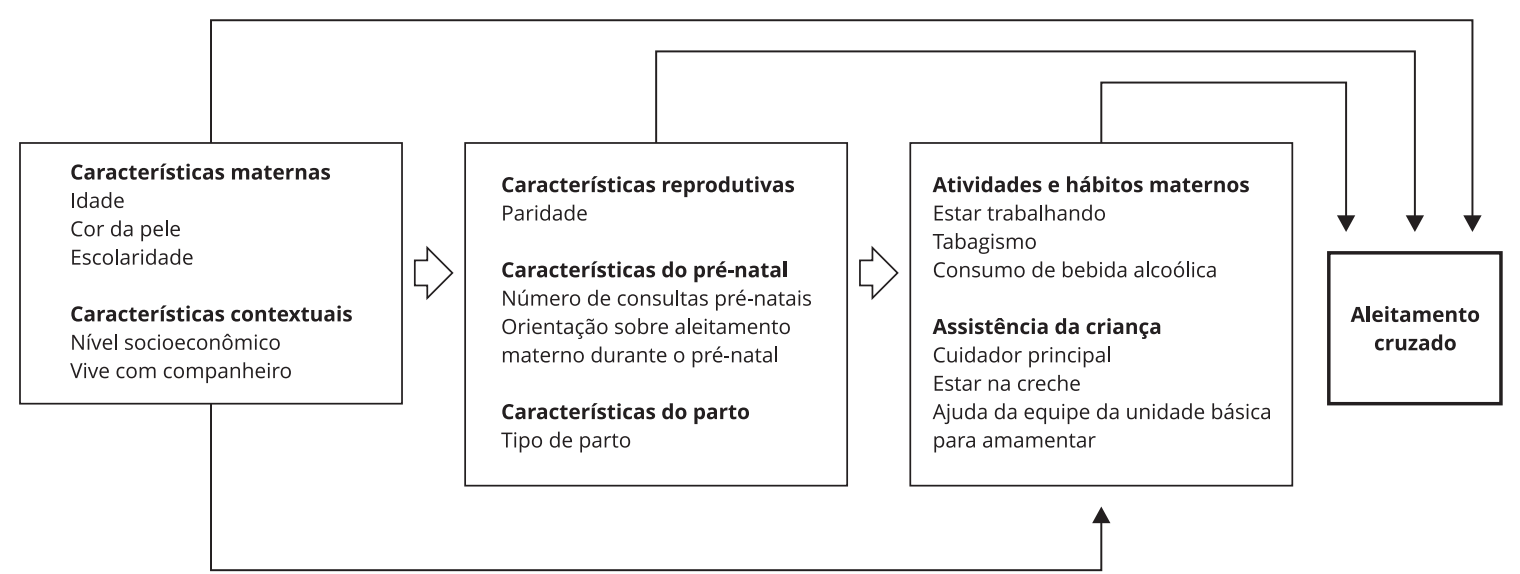




\section{Resultados}

O aleitamento cruzado foi praticado por 43,4\% ( $\mathrm{n}=250)$ das mães da cidade da Região Metropolitana: $14,2 \%$ amamentaram outro(s) bebê(s) que não o seu filho ( $\mathrm{n}=82), 16,3 \%$ tiveram o seu filho amamentado por outra(s) nutriz(es) $(\mathrm{n}=94)$ e $12,8 \%$ praticaram as duas formas de aleitamento cruzado $(\mathrm{n}=$ 74). Na cidade da Região Serrana, a prevalência do aleitamento cruzado foi de $34,5 \%(n=252): 10,5 \%$ amamentaram outro(s) bebê(s) que não o seu filho ( $\mathrm{n}=77)$, 13\% tiveram o seu filho amamentado por outra(s) nutriz(es) $(\mathrm{n}=95)$ e $10,9 \%$ tanto amamentaram como tiveram o seu filho amamentado por outra(s) mulher(es) $(\mathrm{n}=80)$.

Nas duas cidades, a maioria das mães praticou o aleitamento cruzado entre parentes, e a razão mais frequente para a prática foi o cuidado do bebê por nutriz que não a sua mãe (Tabela 1).

Mais de um quinto das mulheres era adolescente, com maior frequência na cidade da Região Metropolitana. A maioria das mães tinha cor de pele não branca, escolaridade inferior ao Ensino Fundamental, pertencia à classe $\mathrm{C}$ e vivia com companheiro (Tabela 2).

Em ambas as cidades, a maioria das mães era multípara. Na cidade da Região Metropolitana, menos de dois terços das mulheres foram acompanhados por seis ou mais consultas de pré-natal, e na cidade da Região Serrana mais de 90\% delas alcançaram este acompanhamento. A grande maioria das mulheres recebeu alguma orientação sobre aleitamento materno no pré-natal e pouco mais da metade das mães tiveram seu filho por parto normal (Tabela 2).

Mais de um quarto das mães trabalhava fora de casa, cerca de um décimo delas fumava e o mesmo percentual ingeria bebida alcoólica. Na cidade da Região Serrana, mais da metade das mães consideravam que a unidade básica de saúde as ajudava a amamentar, já na cidade da Região Metropolitana menos de um terço das mães se sentiu ajudada (Tabela 2).

Na cidade da Região Metropolitana, na análise bivariada, estiveram associadas ao aleitamento cruzado as variáveis distais: idade e cor da pele materna, nível socioeconômico e viver com companheiro; a variável intermediária: paridade; e as variáveis proximais: fumo, uso de bebida alcoólica e trabalho materno atual (Tabela 2). Na cidade da Região Serrana, foram associadas ao aleitamento cruzado as variáveis distais: idade, cor da pele e escolaridade materna, nível socioeconômico e viver com companheiro; as variáveis intermediárias: paridade, número de consultas de pré-natal e orientação sobre amamentação durante o pré-natal; e a variável proximal: uso de bebida alcoólica (Tabela 2).

Tabela 1

Prática de aleitamento cruzado segundo a relação entre as mães envolvidas e motivações em duas cidades do Estado do Rio de Janeiro, Brasil, 2008-2010.

Cidade da Região Metropolitana $(n=586$ )

n

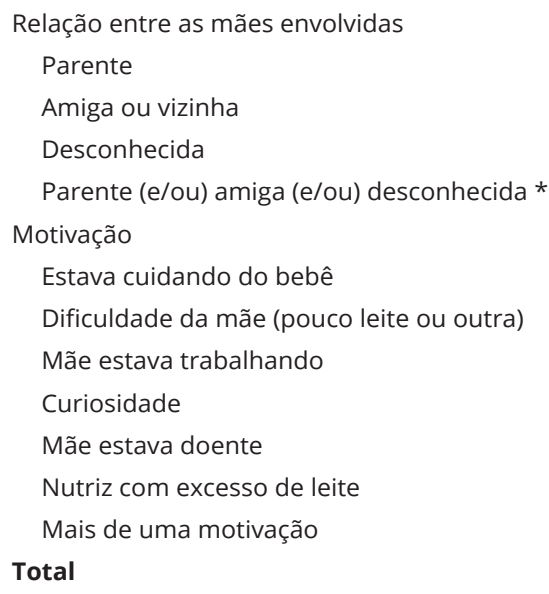

Cidade da Região Serrana $(n=732)$

$\%$

n

$\%$

\begin{tabular}{cccc}
129 & 51,8 & 145 & 57,5 \\
90 & 36,2 & 71 & 28,3 \\
12 & 4,8 & 17 & 6,7 \\
18 & 7,2 & 19 & 7,5 \\
& & & \\
78 & 31,6 & 104 & 41,3 \\
73 & 29,6 & 46 & 18,3 \\
15 & 6,1 & 25 & 9,9 \\
15 & 6,1 & 18 & 7,1 \\
10 & 4,0 & 12 & 4,8 \\
11 & 4,5 & 6 & 2,4 \\
45 & 18,2 & 41 & 16,3 \\
249 & 100,0 & 252 & 100,0 \\
\hline
\end{tabular}

* A prática de aleitamento cruzado aconteceu por duas ou mais das seguintes possibilidades: parente, amiga, e/ou desconhecida. 


\section{Tabela 2}

Prevalência e razão de prevalência (RP) bruta de aleitamento cruzado, segundo características maternas, contextuais, reprodutivas, do pré-natal e do parto; e atividades, hábitos maternos e assistência à criança, em duas cidades do Estado do Rio de Janeiro, Brasil, 2008-2010.

\begin{tabular}{|c|c|c|c|c|c|c|c|c|c|c|}
\hline \multirow[t]{2}{*}{ Características } & \multicolumn{5}{|c|}{ Cidade da Região Metropolitana } & \multicolumn{5}{|c|}{ Cidade da Região Serrana } \\
\hline & \multicolumn{5}{|c|}{$\begin{array}{l}\text { aleitamento } \\
\text { cruzado }\end{array}$} & \multicolumn{5}{|c|}{$\begin{array}{c}\text { aleitamento } \\
\text { cruzado }\end{array}$} \\
\hline \multicolumn{11}{|l|}{ Variáveis distais } \\
\hline \multicolumn{11}{|l|}{ Idade materna (anos) } \\
\hline 20 ou mais & 422 & 73,3 & 40,5 & 1,000 & & 578 & 79,1 & 31,3 & 1,000 & \\
\hline $13-19$ & 154 & 26,7 & 51,3 & 1,266 & 0,021 & 153 & 20,9 & 46,4 & 1,482 & $<0,001$ \\
\hline \multicolumn{11}{|l|}{ Cor da pele materna } \\
\hline Branca & 131 & 22,8 & 38,2 & 1,000 & & 307 & 42,0 & 28,7 & 1,000 & \\
\hline Não branca & 442 & 77,2 & 45,0 & 1,180 & 0,165 & 421 & 58,0 & 38,5 & 1,342 & 0,006 \\
\hline \multicolumn{11}{|l|}{ Escolaridade materna (anos) } \\
\hline 9 completos ou mais & 281 & 48,8 & 41,3 & 1,000 & & 263 & 36,0 & 25,1 & 1,000 & \\
\hline Até 8 completos & 295 & 51,2 & 45,4 & 1,100 & 0,316 & 468 & 64,0 & 39,7 & 1,584 & $<0,001$ \\
\hline \multicolumn{11}{|l|}{ Nível socioeconômico } \\
\hline Classe B & 28 & 4,9 & 21,4 & 1,000 & & 41 & 5,6 & 17,1 & 1,000 & \\
\hline Classe C & 340 & 59,0 & 41,2 & 1,922 & 0,040 & 474 & 64,9 & 30,6 & 1,792 & 0,069 \\
\hline Classes D e E & 208 & 36,1 & 50,0 & 2,333 & 0,004 & 216 & 29,5 & 46,3 & 2,712 & 0,001 \\
\hline \multicolumn{11}{|l|}{ Vive com companheiro } \\
\hline Sim & 468 & 80,0 & 41,7 & 1,000 & & 585 & 79,9 & 32,5 & 1,000 & \\
\hline Não vive ou não tem companheiro & 117 & 20,0 & 50,0 & 1,199 & 0,111 & 147 & 20,1 & 42,2 & 1,296 & 0,028 \\
\hline \multicolumn{11}{|l|}{ Variáveis intermediárias } \\
\hline \multicolumn{11}{|l|}{ Paridade } \\
\hline Multípara & 298 & 51,7 & 38,9 & 1,000 & & 424 & 58,0 & 31,8 & 1,000 & \\
\hline Primípara & 268 & 48,3 & 48,9 & 1,256 & 0,017 & 302 & 42,0 & 37,4 & 1,175 & 0,118 \\
\hline \multicolumn{11}{|l|}{ Número de consultas pré-natais } \\
\hline 6 ou mais & 349 & 60,6 & 43,1 & 1,000 & & 662 & 91,4 & 32,5 & 1,000 & \\
\hline $0-5$ & 227 & 39,4 & 44,5 & 1,032 & 0,745 & 62 & 8,6 & 50,0 & 1,537 & 0,005 \\
\hline \multicolumn{11}{|c|}{$\begin{array}{l}\text { Orientação sobre amamentação durante o } \\
\text { pré-natal }\end{array}$} \\
\hline $\mathrm{Sim}$ & 522 & 92,6 & 42,6 & 1,000 & & 655 & 98,1 & 34,4 & 1,000 & \\
\hline Não & 42 & 7,4 & 52,4 & 1,230 & 0,218 & 13 & 1,9 & 53,8 & 1,568 & 0,144 \\
\hline \multicolumn{11}{|l|}{ Tipo de parto } \\
\hline Normal & 320 & 55,9 & 44,6 & 1,000 & & 380 & 52,3 & 34,3 & 1,000 & \\
\hline Cesariana & 252 & 44,1 & 41,9 & 1,063 & 0,529 & 346 & 47,7 & 34,1 & 1,006 & 0,956 \\
\hline \multicolumn{11}{|l|}{ Variáveis proximais } \\
\hline \multicolumn{11}{|l|}{ Trabalho materno atual } \\
\hline Não & 440 & 75,1 & 44,1 & 1,000 & & 474 & 64,8 & 33,6 & 1,000 & \\
\hline Sim & 133 & 22,7 & 43,1 & 0,977 & 0,835 & 234 & 32,0 & 36,3 & 1,081 & 0,476 \\
\hline Em licença maternidade & 13 & 2,2 & 23,1 & 0,523 & 0,132 & 23 & 3,1 & 30,4 & 0,905 & 0,752 \\
\hline \multicolumn{11}{|l|}{ Tabagismo } \\
\hline Não & 513 & 89,1 & 42,5 & 1,000 & & 633 & 87,3 & 33,7 & 1,000 & \\
\hline Sim & 63 & 10,9 & 53,3 & 1,255 & 0,109 & 92 & 12,7 & 37,0 & 1,097 & 0,539 \\
\hline \multicolumn{11}{|l|}{ Consumo de bebida alcoólica } \\
\hline Não & 503 & 87,3 & 42,9 & 1,000 & & 671 & 92,3 & 33,6 & 1,000 & \\
\hline Socialmente & 41 & 7,1 & 41,5 & 0,966 & 0,854 & 40 & 5,5 & 35,0 & 1,042 & 0,854 \\
\hline Consumo pesado & 32 & 5,6 & 58,6 & 1,365 & 0,098 & 16 & 2,2 & 56,3 & 1,675 & 0,059 \\
\hline Cuidador principal & & & & & & & & & & \\
\hline Mãe & 519 & 88,6 & 43,2 & 1,000 & & 601 & 82,1 & 33,7 & 1,000 & \\
\hline Outro & 67 & 11,4 & 44,8 & 1,036 & 0,809 & 131 & 17,9 & 38,2 & 1,134 & 0,326 \\
\hline
\end{tabular}


Tabela 2 (continuação)

\begin{tabular}{|c|c|c|c|c|c|c|c|c|c|c|}
\hline \multirow[t]{4}{*}{ Características } & \multicolumn{5}{|c|}{ Cidade da Região Metropolitana } & \multicolumn{5}{|c|}{ Cidade da Região Serrana } \\
\hline & $\mathbf{n}$ & $\%$ & $\%$ & $\mathbf{R P}$ & Valor de p & $\mathbf{n}$ & $\%$ & $\%$ & $\mathbf{R P}$ & Valor de $p$ \\
\hline & \multirow{2}{*}{\multicolumn{5}{|c|}{$\begin{array}{c}\text { aleitamento } \\
\text { cruzado }\end{array}$}} & \multirow{2}{*}{\multicolumn{4}{|c|}{$\begin{array}{c}\text { aleitamento } \\
\text { cruzado }\end{array}$}} & \\
\hline & & & & & & & & & & \\
\hline \multicolumn{11}{|c|}{ Variáveis proximais } \\
\hline \multicolumn{11}{|l|}{ Creche } \\
\hline Não & 583 & 99,5 & 43,5 & 1,000 & & 716 & 97,8 & 34,7 & 1,000 & \\
\hline Sim & 3 & 0,5 & 33,3 & 0,767 & 0,724 & 16 & 2,2 & 25,0 & 0,721 & 0,420 \\
\hline \multicolumn{11}{|c|}{ Ajuda da unidade básica para amamentar } \\
\hline Sim & 163 & 28,2 & 40,4 & 1,000 & & 434 & 59,5 & 35,9 & 1,000 & \\
\hline Não & 416 & 71,8 & 44,5 & 1,103 & 0,367 & 295 & 40,5 & 32,7 & 0,908 & 0,360 \\
\hline Total & 586 & 100,0 & 43,4 & & & 732 & 100,0 & 34,5 & & \\
\hline
\end{tabular}

No modelo final, em ambas as cidades, o baixo nível socioeconômico (classes D ou E) foi associado ao aleitamento cruzado. Na cidade da região Serrana, também estiveram diretamente associados ao aleitamento cruzado: ser mãe adolescente, ter sido acompanhada por menos de seis consultas prénatais e ter escolaridade igual ou inferior ao Ensino Fundamental completo. Na cidade da Região Metropolitana, além do nível socioeconômico, apenas a multiparidade foi associada (inversamente) ao desfecho (Tabela 3).

\section{Discussão}

O aleitamento cruzado, embora contraindicado pelo Ministério da Saúde desde 1996, foi bastante frequente em ambas as cidades (43,4\% e 34,5\%), com prevalência superior aos 29,4\% encontrados em estudo transversal conduzido em 2013 com mães de crianças menores de um ano assistidas por unidades básicas do Sistema Único de Saúde (SUS) na cidade do Rio de Janeiro ${ }^{8}$. Essas prevalências relativamente elevadas sugerem que regulamentações ministeriais isoladamente podem não ser suficientes para coibir práticas culturais, uma vez que as decisões maternas em relação à amamentação são construídas com base na interação com a comunidade, com o recém-nascido e em suas atividades diárias 15, e são fortemente influenciadas por familiares, amigos e parceiros 16.

Em ambas as cidades estudadas, o aleitamento cruzado foi mais praticado entre parentes e amigas, o mesmo comportamento encontrado entre mães usuárias de unidades primárias no Município do Rio de Janeiro 8 . Como referido por Thorley 4 , a amamentação cruzada é um compartilhamento informal de leite materno que geralmente ocorre entre mães que possuem relações de confiança, como entre familiares e amigas. Esse comportamento difere de práticas recentes de compartilhamento de leite humano em países de alta renda por contatos via Internet, nas quais a grande maioria das praticantes não se conhece pessoalmente ${ }^{17}$. A principal motivação do aleitamento cruzado foi o cuidado com o bebê por outra nutriz, indicando que se trata de um comportamento de ajuda mútua 4 .

Embora a segunda razão alegada nas duas cidades tenha sido a dificuldade da mãe em amamentar, na cidade da Região Metropolitana, de piores indicadores socioeconômicos e assistência à saúde mais precária, esta motivação foi ainda mais frequente. O fator associado com maior intensidade à amamentação cruzada, e o único comum às duas cidades, foi o baixo nível socioeconômico. Oliveira et al. 18 observaram que, quanto piores as condições de vida de uma família, maiores as chances de interrupção precoce do aleitamento exclusivo ou predominante e introdução precoce de alimentos. Portanto, famílias de menores níveis socioeconômicos têm menor aderência às práticas de aleitamento materno recomendadas pelo Ministério da Saúde, possivelmente por disporem de menor acesso à informação, o que provavelmente contribui para a prática do aleitamento cruzado.

As diferenças no perfil das duas cidades foram refletidas em diferenças nos fatores associados ao desfecho: comparada à cidade da Região Serrana, a cidade da Região Metropolitana conta com uma 


\section{Tabela 3}

Fatores associados ao aleitamento cruzado em duas cidades do Estado do Rio de Janeiro, Brasil, 2008-2010.

\begin{tabular}{|c|c|c|c|c|c|c|}
\hline \multirow[t]{2}{*}{ Características } & \multicolumn{3}{|c|}{$\begin{array}{c}\text { Cidade da Região Metropolita } \\
\text { Modelo final }\end{array}$} & \multicolumn{3}{|c|}{$\begin{array}{c}\text { Cidade da Região Serrana } \\
\text { Modelo final }\end{array}$} \\
\hline & $\mathbf{R P} *$ & Valor de $p$ & IC95\% & $\mathbf{R P}$ * & Valor de $p$ & IC95\% \\
\hline \multicolumn{7}{|l|}{ Idade da mãe (anos) } \\
\hline 20 ou mais & - & - & - & 1,000 & & \\
\hline $13-19$ & & & & 1,419 & 0,001 & $1,146-1,756$ \\
\hline \multicolumn{7}{|l|}{ Escolaridade (anos) } \\
\hline 9 ou mais & - & - & - & 1,000 & & \\
\hline Até 8 completos & & & & 1,340 & 0,024 & $1,118-1,828$ \\
\hline \multicolumn{7}{|c|}{ Nível socioeconômico } \\
\hline Classe B & 1,000 & & & 1,000 & & \\
\hline Classe C & 1,939 & 0,066 & $0,937-3,929$ & 1,748 & 0,145 & $0,824-3,704$ \\
\hline Classes D e E & 2,382 & 0,016 & $1,175-4,830$ & 2,431 & 0,024 & $1,125-5,251$ \\
\hline \multicolumn{7}{|l|}{ Paridade } \\
\hline Primípara & 1,000 & & & - & - & - \\
\hline Multípara & 0,780 & 0,009 & $0,648-0,940$ & & & \\
\hline \multicolumn{7}{|c|}{$\begin{array}{l}\text { Número de consultas pré- } \\
\text { natais }\end{array}$} \\
\hline 6 ou mais & - & - & - & 1,000 & & \\
\hline $0-5$ & - & - & - & 1,327 & 0,046 & $1,005-1,753$ \\
\hline
\end{tabular}

IC95\%: intervalo de 95\% de confiança.

* Razão de prevalência ajustada.

população socioeconomicamente mais homogênea 10, o que provavelmente contribuiu para que poucas variáveis captassem diferenças em relação à prática do aleitamento cruzado nesta cidade.

Na cidade da Região Serrana, as mulheres com menor nível de escolaridade tiveram prevalência relativamente maior de aleitamento cruzado do que aquelas com mais escolaridade. Além disso, mulheres com número insuficiente de consultas pré-natais apresentaram uma prevalência 33\% superior do desfecho. Boccolini et al. ${ }^{19}$, em revisão sistemática, observaram que, em 11 de 29 estudos, mães com maior escolaridade tiveram maior prevalência ou duração de aleitamento materno exclusivo, o que pode estar relacionado ao seu conhecimento sobre a importância deste tipo de aleitamento, conhecimento este que pode ser trabalhado durante o cuidado pré-natal. Mães menos informadas e orientadas no pré-natal podem ter mais dificuldades para amamentar. Como observado por Diehl \& Anton 20 , quando as mães não encontram soluções para as suas dificuldades nas informações disponíveis, utilizam as experiências prévias e as ferramentas de que dispõem, como a introdução precoce de alimentos, de leites infantis e, possivelmente, a prática do aleitamento cruzado.

Ainda na cidade da Região Serrana, dentre as mães adolescentes a prevalência de aleitamento cruzado foi $42 \%$ superior, quando comparadas às mães adultas. Considerando que o aleitamento cruzado é uma prática social e de ajuda mútua, não é de estranhar que ao se depararem com os desafios da gravidez e da maternidade, mães adolescentes busquem redes de apoio informal nas quais recebem e proporcionam apoio 21 .

Na cidade da Região Metropolitana, além do baixo nível socioeconômico, apenas a multiparidade esteve associada ao desfecho: mulheres que tiveram gestações anteriores praticaram menos o aleitamento cruzado. De acordo com Vieira et al. 22, mães multíparas apresentam uma maior prevalência de aleitamento materno e de aleitamento materno exclusivo. Os autores acreditam que fatores culturais que favorecem a introdução precoce de alimentos, como crenças e hábitos passados entre gerações, tenham maior peso no primeiro parto. Além disso, num estudo qualitativo ${ }^{23}$, a falta de experiência prévia com a amamentação foi relatada como justificativa para a interrupção do aleitamento materno, 
pelas dificuldades com as quais a mãe sem experiência não consegue lidar. Prática cultural enraizada, é possível que o aleitamento cruzado tenha sido mais praticado pelas mães primíparas do presente estudo frente às dificuldades e necessidade de apoio destas mães para amamentar.

Este trabalho apresenta limitações. Não há informação sobre o momento de início e de término da prática do aleitamento cruzado, o que impossibilita o estabelecimento de relação causal entre o desfecho e a maior parte das variáveis proximais investigadas. Além disso, há possibilidade de viés de informação, pois não ocorreu observação direta da mesma pela equipe de pesquisa, e a única fonte de informação disponível sobre o aleitamento cruzado foi a entrevista materna. Todavia, pode-se assumir que esse viés de informação não seja diferencial, ou seja, a medida de associação com o desfecho não teve tendência a sub ou superestimação.

Conclui-se que o aleitamento cruzado foi mais praticado pelas populações mais vulneráveis: mães adolescentes, com baixa escolaridade, com menor nível socioeconômico, com número insuficiente de consultas pré-natais e primíparas. Além disso, o trabalho sinaliza um distanciamento entre o posicionamento de autoridades de saúde e as práticas que de fato ocorrem entre as mães brasileiras.

Recomenda-se que mais estudos sejam conduzidos acerca do aleitamento cruzado, explorando aspectos como a sua frequência e duração, sua prevalência entre mães usuárias da rede privada de saúde, bem como investigando os fatores associados à prática em outros contextos. É necessário, inclusive, avaliar os conhecimentos e práticas profissionais relativas ao tema.

O aleitamento cruzado deve receber mais atenção dos profissionais e instituições de saúde, pois está evidente que o posicionamento atual frente ao tema não está refletindo nas escolhas de parte significativa das mães, e é questionável. Essa prática cultural pode estar beneficiando mães que se apoiam mutuamente e bebês que recebem leite humano. Além disso, a prevalência de HIV em gestantes no contexto brasileiro é baixa $(0,4 \%) 24$, e a proporção de mães não testadas para o HIV na gestação e no momento do parto foi inferior a $1 \%$ em estudo recente 25 . Portanto, amplo debate sobre o aleitamento cruzado deve ser promovido envolvendo o meio acadêmico, o setor saúde e a sociedade em geral, e investigações devem avaliar a relação entre os benefícios e os riscos inerentes ao aleitamento cruzado.

\section{Colaboradores}

M. P. von Seehausen participou da concepção geral do estudo e foi a responsável principal pela análise e interpretação dos dados, redação do artigo e aprovação final da versão a ser publicada. M. I. C. Oliveira participou da concepção geral do estudo, da análise e interpretação dos dados, redação do artigo e aprovação final da versão a ser publicada. C. S. Boccolini participou da concepção geral do estudo, da análise e interpretação dos dados e aprovação final da versão a ser publicada. $M$. C. Leal foi a responsável principal pela concepção geral do estudo e participou da aprovação final da versão a ser publicada.

\section{Agradecimentos}

Agradecemos a todos os entrevistadores que trabalharam nesta pesquisa e a todas as mães que gentilmente concederam as entrevistas. À Faperj (E-26/152.591/2006) e ao CNPq (409805/2008-8) pelo financiamento.

\section{Referências}

1. Obladen M. Regulated wet nursing: managed care or organized crime? Neonatology 2012; 102:222-8

2. Koutsoukos SSM. 'Amas mercenárias': o discurso dos doutores em medicina e os retratos de amas - Brasil, segunda metade do Século XIX. Hist Ciênc Saúde-Manguinhos 2009; 16:305-24.

3. Krantz JZ, Kupper NS. Cross-nursing: wet nursing in a contemporary context. Pediatrics 1981; 67:715-17.

4. Thorley V. Sharing breastmilk: wet nursing, cross feeding, and milk donations. Breastfeed Rev 2008; 16:25-9.

5. La Leche League Internacional. Wet nursing and cross nursing. http://www.lalecheleague. org/llleaderweb/lv/lvjulaug95p53.html (acessado em 17/Fev/2016).

6. Ministério da Saúde. Portaria no 2.415 de 12 de dezembro de 1996. Dispõe sobre medidas para prevenção da contaminação pelo HIV pelo aleitamento materno. Diário Oficial da União 1996; 19 dez. 
7. Nogueira CMR. Conhecimento sobre aleitamento materno de parturientes e prática de aleitamento cruzado na Unidade Hospitalar e Maternidade Venâncio Raimundo de Souza Horizonte - Ceará [Dissertação de Mestrado]. Rio de Janeiro: Escola Nacional de Saúde Pública Sergio Arouca, Fundação Oswaldo Cruz; 2008.

8. von Seehausen MP, Oliveira MIC, Boccolini CS. Fatores associados ao aleitamento cruzado. Ciênc Saúde Coletiva 2017; 22:1673-82.

9. Vieira ACJ, Amaral DF, Pedrosa KJ, Ichisato SMT. Conhecimento das puérperas sobre aleitamento cruzado. Revista do Centro Universitário Barão de Mauá 2001; 1:1-12.

10. Leal MC, Pereira AP, Lamarca GA, Vettore MV. The relationship between social capital, social support and the adequate use of prenatal care. Cad Saúde Pública 2011; 27 Suppl 2:S237-53.

11. Associação Brasileira de Empresas de Pesquisa. Códigos e guias: critério de classificação econômica Brasil (CCEB). http://www.abep.org (acessado em 22/Set/2015).

12. Instituto Brasileiro de Geografia e Estatística. Pesquisa das características étnico-raciais da População - PcerP. Brasília: Ministério do Planejamento, Orçamento e Gestão; 2012.

13. Coutinho LMS, Scazufca M, Menezes PR. Métodos para estimar razão de prevalência em estudos de corte transversal. Rev Saúde Pública 2008; 42:992-8.

14. Victora CG, Huttly SR, Fuchs SC, Olinto MT. The role of conceptual frameworks in epidemiological analysis: a hierarchical approach. Int J Epidemiol 1997; 26:224-7.

15. Sertório SC, Silva IA. The symbolic and utilitarian facets of pacifiers according to mothers. Rev Saúde Pública 2005; 39:156-62.

16. Olson BH, Horodynski MA, Brophy-Herb H, Iwanski KC. Health professionals' perspectives on the infant feeding practices of low income mothers. Matern Child Health J 2010; 14:75-85.
17. Reyes-Foster BM, Carter SK, Hinojosa MS Milk sharing in practice: a descriptive analysis of peer breastmilk sharing. Breastfeed Med 2015; 10:263-9.

18. Oliveira LP, Assis AM, Gomes GS, Prado MS, Barreto ML. Duração do aleitamento materno, regime alimentar e fatores associados segundo condições de vida em Salvador, Bahia, Brasil. Cad Saúde Pública 2005; 21:1519-30.

19. Boccolini CS, Carvalho ML, Oliveira MI. Factors associated with exclusive breastfeeding in the first six months of life in Brazil: a systematic review. Rev Saúde Pública 2015; 49:91.

20. Diehl JP, Anton MC. Fatores emocionais associados ao aleitamento materno exclusivo e sua interrupção precoce: um estudo qualitativo. Aletheia 2011; (34):47-60.

21. Stevenson W, Maton KI, Teti DM. Social support, relationship quality, and well-being among pregnant adolescents. J Adolesc 1999; 22:109-21.

22. Vieira GO, Almeida JAG, Silva LR, Cabral VA, Santana PVN. Fatores associados ao aleitamento materno e desmame em Feira de Santana, Bahia. Rev Bras Saúde Matern Infant 2004; 4:143-50.

23. Ramos CV, Almeida JAG. Alegações maternas para o desmame: estudo qualitativo. J Pediatr (Rio J.) 2003; 79:385-90.

24. Domingues RMSM, Szwarcwald CL, Souza Jr. PRB, Leal MC. Prenatal testing and prevalence of HIV infection during pregnancy: data from the "Birth in Brazil" study, a national hospitalbased study. BMC Infect Dis 2015; 15:100.

25. Gomes DM, Oliveira MIC, Fonseca SC. Avaliação da testagem anti-HIV no pré-natal e na assistência ao parto no Rio de Janeiro, Brasil. Rev Bras Saúde Matern Infant 2015; 15:413-23. 


\section{Abstract}

The objective was to estimate the prevalence of cross-nursing in two Brazilian cities and analyze factors associated with the practice. This was a cross-sectional study nested in a cohort of pregnant women recruited at public health services that offered prenatal care in two medium-sized cities in the State of Rio de Janeiro: one in Greater Metropolitan Rio and the other in the Mountainous Region, conducted from 2008 to 2010. The current study included women interviewed when their infants were six months old. Adjusted prevalence ratios were obtained with a Poisson regression model, and exposure variables that reached $p$ $\leq 0.05$ comprised the final model. Cross-nursing was practiced by $43.4 \%$ of the mothers in Greater Metropolitan Rio de Janeiro and 34.5\% in Mountainous Region. Low socioeconomic status was associated with cross-nursing in both cities. In addition, in Mountainous Region the outcome was directly associated with adolescent motherhood, maternal schooling less than or equal to complete primary, and fewer than six prenatal visits. In Greater Metropolitan Rio de Janeiro, in addition to socioeconomic status, only multiparity was associated (inversely) with cross-nursing. Crossnursing, although contraindicated by the Brazilian Ministry of Health, showed high prevalence among the mothers in the sample and was more frequent among the more vulnerable population groups.

Breast Feeding; Primary Health Care; Child Health

\section{Resumen}

El objetivo fue estimar la prevalencia de la lactancia materna cruzada en dos ciudades brasileñas y analizar los factores asociados a la práctica. Estudio transversal basado en una cohorte de gestantes, reclutadas en los servicios públicos de salud que ofrecían atención pre-natal, en dos ciudades de tamaño medio del Estado de Río de Janeiro: de la Región Metropolitana y de la Región Serrana, dirigido entre 2008 y 2010. El presente trabajo incluye a todas las mujeres entrevistadas a los seis meses de vida de sus bebés. Las razones de prevalencia ajustadas se obtuvieron mediante un modelo de regresión de Poisson y las variables de exposición que alcanzaron $p \leq 0,05$ compusieron el modelo final. La lactancia materna cruzada se practicó por parte de un 43,4\% de las madres en la región metropolitana y por un 34,5\% de ellas en la Región Serrana. El bajo nivel socioeconómico estuvo asociado a la lactancia materna cruzada en las dos ciudades. Asimismo, en la región serrana se asociaron directamente al resultado de ser madre adolescente, tener escolaridad igual o inferior a la Enseñanza Fundamental completa, y haber realizado menos de seis consultas pre-natales. En la región metropolitana, además del nivel socioeconómico, solamente la multiparidad fue asociada (inversamente) a la lactancia materna cruzada. La lactancia materna cruzada, pese a que está contraindicada por el Ministerio de Salud, presentó una alta prevalencia entre las madres entrevistadas y fue más practicada por las poblaciones más vulnerables.

Lactancia Materna; Atención Primaria de Salud; Salud del Niño
Recebido em 08/Mar/2016

Versão final reapresentada em 23/Jun/2016 Aprovado em 13/Jul/2016 